\title{
New Book Reviews Editor
}

Let me make my first official comment as Book Reviews Editor to thank my predecessor, Dr Karen Windle, for her work with the Journal.

I join PHCR\&D at an exciting time when Primary Care is being recognised as playing an ever more important role in the delivery of the national and international health care agendas. The Journal has a history of attracting contributions from a wide range of health care professionals.

My goal is to take forward the Book Review section of the Journal by publishing lively, balanced, informative and responsible provocative criticisms on the widest possible range of health-related books. This will provide a snapshot of health-related issues as they exist at a particular time through timely publications of reviews relevant to our readers. In order to achieve my goal I shall call on the expertise of a number of professionals and researchers to provide a wide and balanced view of the literature presently available in book form and pertinent to the primary health care agenda - in its widest form.

There is no preferred length of review although a typical review might be between 1000 and 1500 words depending on the book in question. Unusually, interesting and unconventional contributions will be considered for publication. Responding promptly is vital! If you would like to undertake a book review please contact me with an indication of your areas of research interest and the number of reviews per year you could undertake.

I look forward to working with many of you over the next few years.

Maxine Offredy University of Hertfordshire Hatfield, UK Email: m.v.offredy@herts.ac.uk 